\title{
Premedication Methods in Nasal Endoscopy: A Prospective, Randomized, Double-Blind Study
}

\author{
Mehmet IIhan Șahin ${ }^{1}$ Kerem Kökoğlu' · Șafak Güleç² $\cdot$ İbrahim Ketenci ${ }^{1}$ Yașar Ünlü ${ }^{1}$ \\ ${ }^{l}$ Department of Otolaryngology, Erciyes University Medical School, Kayseri; ${ }^{2}$ Department of Otolaryngology, Akdağmadeni State Hospital, \\ Yozgat, Turkey
}

Objectives. To identify the optimal pharmacological method of preparing patients for nasal endoscopy.

Methods. Twenty healthy volunteers were enrolled in this prospective, randomized, double-blind study. Four types of medications were applied in their nostrils with binary combinations of spray bottles on four different days in a random order: placebo (normal saline [NS]+NS), decongestant (NS+oxymetazoline), anesthetic (NS+lidocaine), and decongestant plus anesthetic (oxymetazoline+lidocaine). Rigid nasal endoscopy was performed 10 minutes after spray application. The volunteers evaluated the discomfort caused by each spray application, and nasal pain scores due to the passage of the endoscope. The physicians quantified nasal decongestion using a visual analogue scale. Endoscopy duration as well as pulse and mean blood pressure (MBP) before spray application, 10 minutes after the application, and immediately after endoscopic examination were also recorded.

Results. The discomfort caused by lidocaine was significantly higher than that caused by the other sprays $(P<0.001)$. The lowest pain score related to endoscopy was obtained for oxymetazoline+lidocaine $(P<0.001)$. Nasal decongestion was best achieved with NS+oxymetazoline $(P<0.001)$. Endoscopy duration was the shortest for oxymetazoline+ lidocaine $(P<0.05)$. Statistically significant MBP changes were only seen with the application of NS+oxymetazoline $(P<0.05)$. However, neither MBP nor pulse rate change was significant clinically.

Conclusion. Application of decongestant and anesthetic sprays together seems to be the best method of pharmacological preparation of patients for nasal endoscopy.

Keywords. Anesthetics; Nasal Decongestants; Lidocaine; Oxymetazoline; Endoscopy

\section{INTRODUCTION}

Nasal endoscopy (NE) is a widespread and valuable examination method in otolaryngology practice [1]. NE allows the visualization of the posterior and superior parts of the nasal airway, which cannot be seen by other means, e.g., anterior rhinoscopy. $\mathrm{NE}$ is usually accomplished with a rigid endoscope, but a flexible endoscope is preferred in patients with severe nasal septal deviation [2]. For the patients, NE is a painful and uncomfort-

- Received April 27, 2016

Revised June 7, 2016

Accepted June 17, 2016

- Corresponding author: Mehmet Illhan Şahin

Department of Otolaryngology, Erciyes University Medical School,Talas

Yolu, Kayseri 38039, Turkey

Tel: +90-352-207-6666, Fax: +90-352-437-5273

E-mail: misahin@erciyes.edu.tr able procedure [3]; for the physicians, the disadvantages are prolonged procedure duration and limited view of nasal passages due to hypertrophic and swollen turbinates. In addition, some physiological changes such as acceleration of pulse rate and increase of blood pressure have been associated with NE [4].

As a preparation for NE, a local anesthetic and/or a decongestant are usually applied to nostrils in order to alleviate the nasal pain, to widen the field of view, and to shorten the duration of the examination. However, the benefits of local anesthetics are controversial, partly because they cause discomfort to patients because of the bad taste and smell $[5,6]$. Furthermore, there is always a suspicion that local decongestants may cause hypertension and reflex bradycardia [7]. Some authors recommend no medication before NE on the strength of placebo-controlled studies [8-11]. Other authors recommend only decongestant or decongestant-local anesthetic combination applications [12-14].

Copyright ( $) 2017$ by Korean Society of Otorhinolaryngology-Head and Neck Surgery.

This is an open-access article distributed under the terms of the Creative Commons Attribution Non-Commercial License (http://creativecommons.org/licenses/by-nc/4.0)

which permits unrestricted non-commercial use, distribution, and reproduction in any medium, provided the original work is properly cited. 
Nevertheless, there is no consensus about the best method of preparing the patients for NE $[1,3,10,15,16]$.

In this study, we aimed to identify the optimal pharmacological method of preparing the patients for NE while considering both the clinicians' needs and the patients' comfort and physiological stability.

\section{MATERIALS AND METHODS}

This prospective, randomized, double-blind study was approved by the local ethics committee. After obtaining informed consents, 20 healthy volunteers were enrolled. Exclusion criteria were asthma, cardiovascular disease, rhinitis, severe septal deviation, and a history of nasal endoscopic examination.

Four spray bottles of similar external appearance were prepared and numbered; two of the bottles contained normal saline (NS; $0.9 \%$ sodium chloride), one contained Iliadin $(0.05 \%$ oxymetazoline; Merck, Darmstadt, Germany), and one contained Xylocaine (10\% lidocaine; AstraZeneca, Södertälje, Sweden). The nurse who prepared the bottles was the only person who was aware of the contents of the bottles.

Four binary combinations of sprays were applied in each subject's nostrils in a random order on four different days. In each day, two puffs (one puff per bottle; $0.1 \mathrm{mg}$ oxymetazoline for Iliadin, $20 \mathrm{mg}$ lidocaine for Xylocaine) were applied to both nostrils by the same clinical nurse. All four combinations were applied to each subject to reduce the bias of subjective evaluation and eliminate individual differences. The application and evaluation processes were identical for each combination (Fig. 1).

\section{Spray combinations}

The four binary combinations of sprays were as follows: placebo (NS+NS), decongestant (NS+oxymetazoline), anesthetic (NS+ lidocaine), decongestant plus anesthetic (oxymetazoline+lidocaine).

\section{Nasal endoscopy}

All endoscopies were performed 10 minutes after medications by the same experienced physician who was blinded to the administered drugs using a 4.0-mm, 0-degree rigid endoscope (Karl

\section{H I $G$ G H L I I G H T S}

- Lidocaine discomforted the patients more than placebo.

- Oxymetazoline caused the best and lidocaine caused the worst decongestion score.

- The lowest pain score related to endoscopy was obtained for oxymetazoline plus lidocaine.

- Decongestant plus anesthetic spray seems to be the best premedication in nasal endoscopy.

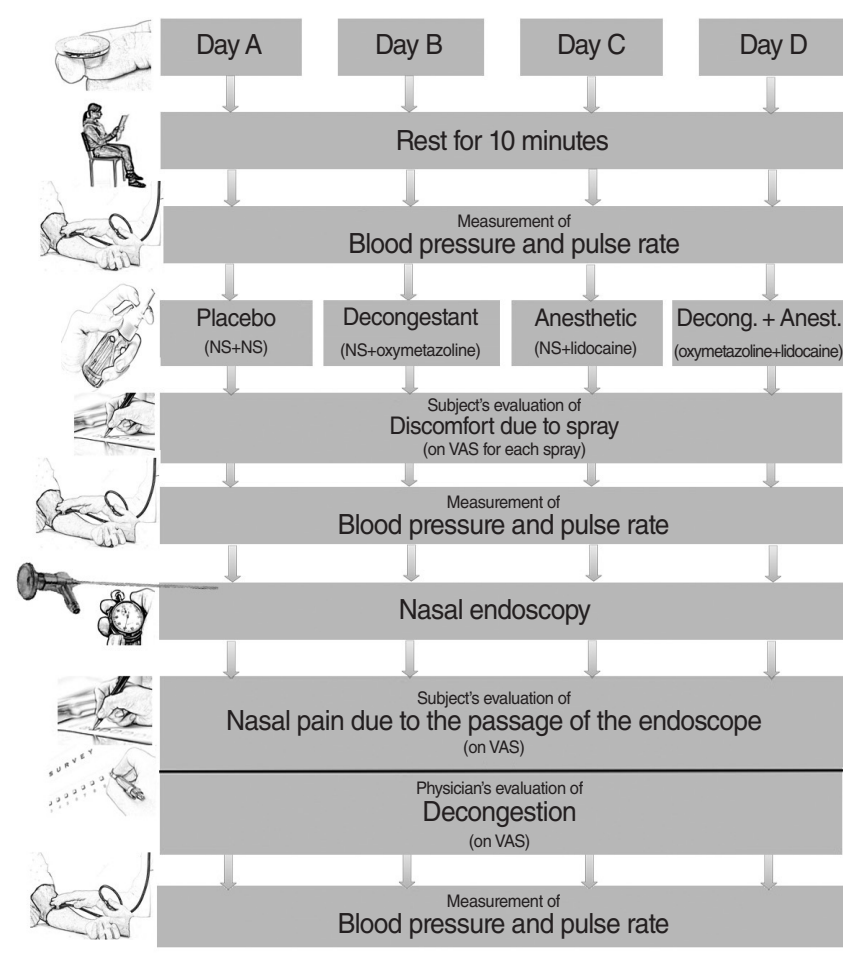

Fig. 1. Flowchart of the method. NS, normal saline; Decong., decongestant; Anest., anesthetic; VAS, visual analog scale.

STORZ GmbH \& Co. KG, Tuttlingen, Germany). In each endoscopy, choana, middle meatus, sphenoethmoidal recess, and superior concha were observed in both nasal passages. The nurse recorded the durations of the endoscopic examinations as seconds.

\section{Clinical evaluations}

Evaluation of discomfort due to the sprays

Shortly after spraying each bottle, subjects were asked to indicate their degree of discomfort on a visual analogue scale (VAS) (1: no discomfort, 10: utmost discomfort). For each combination, two independent scores were obtained from each subject (Fig. 2).

\section{Evaluation of nasal pain due to the passage of the endoscope}

Shortly after endoscopy, subjects were asked to indicate their degree of pain on aVAS (1: no pain, 10: unbearable pain) (Fig. 2).

\section{Evaluation of decongestion (field of view)}

Shortly after endoscopy, the endoscopist was asked to indicate the degree of nasal decongestion on a VAS (1: impossible to pass the endoscope, 10: excellent field of view) (Fig. 2).

\section{Measurement of blood pressure and pulse}

The blood pressure and pulse were measured thrice at each application: just before spray application (10 minutes after a rest); 10 minutes after spray application (just before the endoscopy); 


\begin{tabular}{|c|c|c|c|c|c|c|c|c|c|}
\hline \multicolumn{10}{|c|}{ Volunteer's survey } \\
\hline \multicolumn{4}{|c|}{$\begin{array}{l}\text { Discomfort due to the 1. spray } \\
1 \text { : No discomfort }\end{array}$} & \multicolumn{3}{|c|}{ Spray \# ...... (nurse fills) } & \multicolumn{3}{|c|}{ 10: Utmost discomfort } \\
\hline 1 & 2 & 3 & 4 & 5 & 6 & 7 & 8 & 9 & 10 \\
\hline \multicolumn{4}{|c|}{$\begin{array}{l}\text { Discomfort due to the } 2 \text {. spray } \\
\text { 1: No discomfort }\end{array}$} & \multicolumn{3}{|c|}{ Spray \# ...... (nurse fills) } & \multicolumn{3}{|c|}{ 10: Utmost discomfort } \\
\hline 1 & 2 & 3 & 4 & 5 & 6 & 7 & 8 & 9 & 10 \\
\hline \multicolumn{7}{|c|}{$\begin{array}{l}\text { Nasal pain due to the passage of the endoscope } \\
1 \text { : No pain }\end{array}$} & \multicolumn{3}{|c|}{ 10: Unbeatable pain } \\
\hline 1 & 2 & 3 & 4 & 5 & 6 & 7 & 8 & 9 & 10 \\
\hline \multicolumn{10}{|c|}{ Physician's survey } \\
\hline \multicolumn{7}{|c|}{$\begin{array}{l}\text { Decongestion (field of view) } \\
\text { 1: Impossible to pass the endoscope }\end{array}$} & \multicolumn{3}{|c|}{ 10: Excellent field of view } \\
\hline 1 & 2 & 3 & 4 & 5 & 6 & 7 & 8 & 9 & 10 \\
\hline
\end{tabular}

Fig. 2. Visual analog scale (VAS) questionnaires.

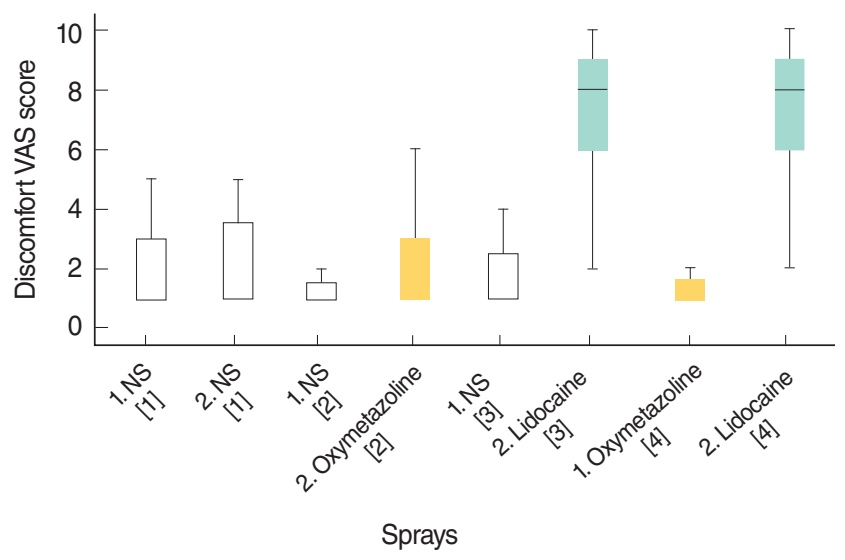

Fig. 3. Distribution of visual analog scale (VAS) scores of discomfort due to the spray. Numbers in brackets show the type of the spray combination. [1] placebo; [2] decongestant; [3] anesthetic; [4] decongestant plus anesthetic. Numbers before the names of the sprays show the order in that combination. NS, normal saline.

and immediately after the endoscopy. The same nurse did all the measurements. Blood pressure was measured with the same calibrated manometer and from the same arm of each subject. Mean blood pressures (MBP) in mmHg and change between the measurements for each application were recorded. Pulse rate was detected by palpating the radial artery, and recorded as beats per minute (BPM).

\section{Statistical analysis}

For statistical analyses, SigmaStat 3.5 (Systat Software, Inc.; http://www.sigmaplot.co.uk/) software was used. A ShapiroWilk test was used to test the normality of data distribution. Normally distributed data are presented as means \pm standard deviation (SD). An analysis of variance test was used for repeated measures. Pairwise comparisons were performed by using the post-hoc parametric Student-Newman-Keuls method. The

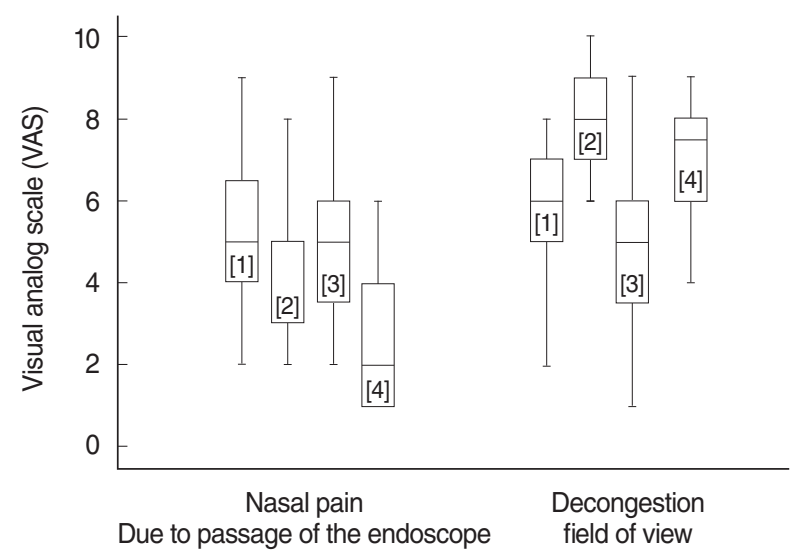

Fig. 4. Distribution of visual analog scale (VAS) scores of nasal pain and decongestion. Numbers in brackets show the type of the spray combination. [1] placebo (NS+NS); [2] decongestant (NS+oxymetazoline); [3] anesthetic (NS+lidocaine); [4] decongestant plus anesthetic (oxymetazoline+lidocaine). NS, normal saline.

data, which was not normally distributed, are presented as medians (range), and a Friedman test was used for their analysis. Pairwise comparisons were performed by the nonparametric Student-Newman-Keuls method. A $P$-value less than 0.05 was considered statistically significant.

\section{RESULTS}

Twenty volunteers ( 12 men, 8 women) aged $27 \pm 7$ years (range, 19 to 50 years) were enrolled in this study.

The discomfort caused by lidocaine was significantly higher than that caused by the other sprays $(P<0.001)$. There were no differences in discomfort between oxymetazoline and NS $(P>$ 0.05) (Fig. 3).

Nasal pain scores due to the passage of the endoscope were significantly different between spray combinations $(P<0.001)$. The lowest pain score was obtained for oxymetazoline+ lidocaine $(P<0.05)$ (Fig. 4). There were no differences in pain between NS+lidocaine and NS+NS $(P>0.05)$.

Nasal decongestion scores were significantly different between spray combinations $(P<0.001)$. Nasal decongestion was best achieved with NS+oxymetazoline $(P<0.001)$, whereas NS+lidocaine had the least effect (Fig. 4). Nasal decongestion scores were significantly higher for NS+oxymetazoline than for oxymetazoline+lidocaine $(P<0.05)$ (Fig. 4).

Duration of endoscopy was significantly shorter for oxymetazoline+lidocaine compared to the other spray combinations $(P<$ $0.05)$, and there were no differences between the other combinations $(P>0.05)$ (Fig. 5).

MBP values were increased significantly by $4.05 \pm 3.31$ mmHg after NS+oxymetazoline application $(P<0.05)$ (Fig. 6). However, neither this change nor the changes of MBP with the 


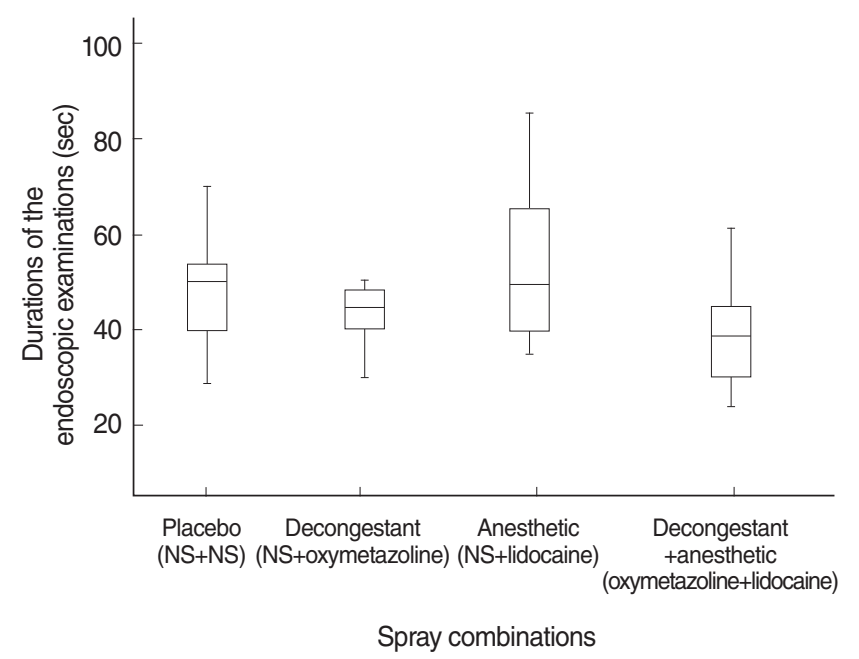

Fig. 5. Distribution of durations of the endoscopic examinations. NS, normal saline.

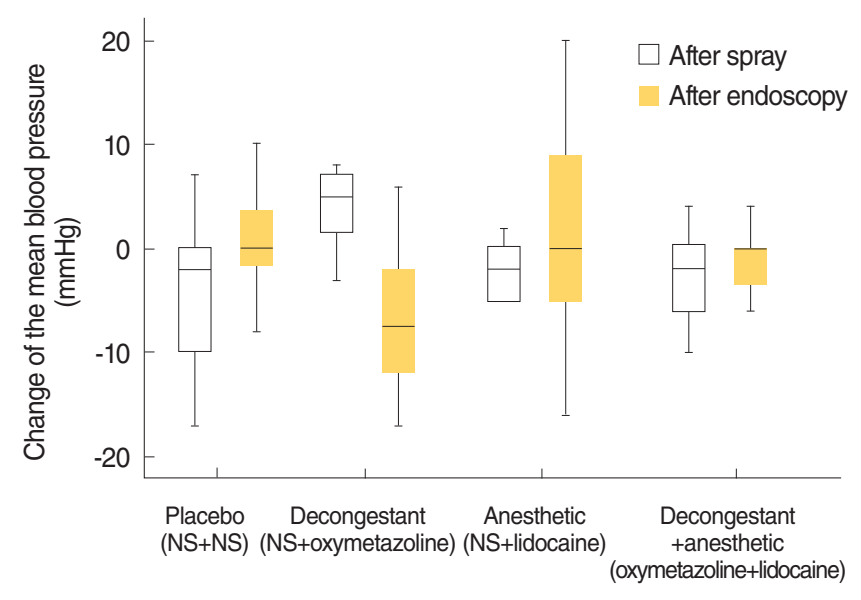

Spray combinations

Fig. 6. Distribution of changes of the mean blood pressure from the beginning to 10 minutes after spray application and from that point to just after endoscopy. NS, normal saline.

other combinations were clinically meaningful. There were no significant changes in pulse rates, neither statistically nor clinically $(P>0.05)$ (Fig. 7). After endoscopy, MBP values were decreased significantly by $7.05 \pm 6.41 \mathrm{mmHg}$, and pulse rates were decreased significantly by $8.65 \pm 7.82 \mathrm{BPM}$ for NS+oxymetazoline $(P<0.001)$. There were no differences in MBP or pulse rate for the other spray combinations (Figs. 6, 7).

\section{DISCUSSION}

In this study, we compared the effects of four different spray combinations as premedication in nasal endoscopy. We confirmed that lidocaine spray is very uncomfortable for patients. Besides, it does not reduce pain during endoscopy, and it nar-

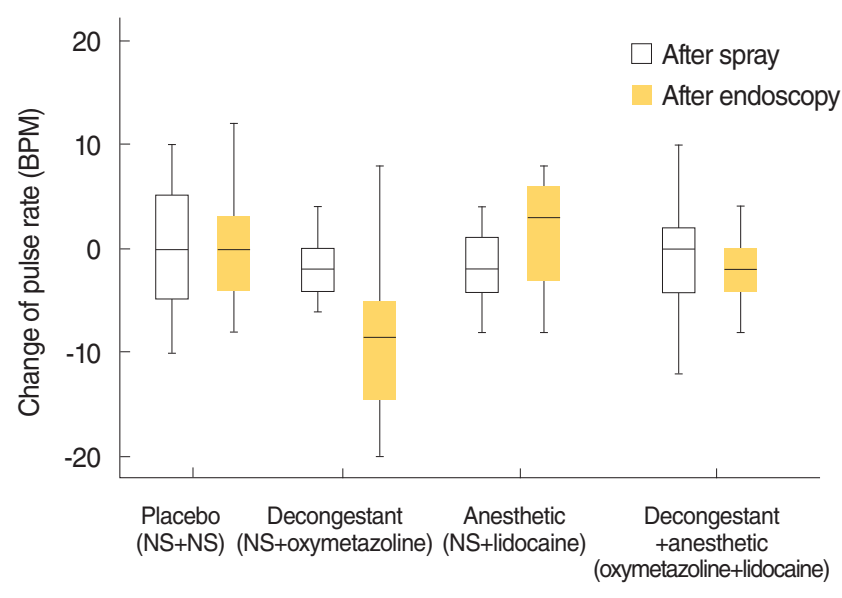

Spray combinations

Fig. 7. Distribution of changes of the pulse rate from the beginning to 10 minutes after spray application and from that point to just after endoscopy. NS, normal saline; BPM, beats per minute.

rows the field of view in nasal passages and prolongs the duration of endoscopy. According to our results, oxymetazoline is comfortable for patients. It reduces pain during endoscopy and provides a wide field of view for the endoscopist. However, it causes a slight increase in arterial blood pressure. When oxymetazoline and lidocaine are used together, pain due to endoscopy is reduced significantly, the endoscopist's field of view is wide, and the duration of endoscopy is shortened.

Premedication in NE is typically applied with sprays or cotton pledgets. Although application of cotton pledgets is an effective method, it is irritating for patients $[17,18]$. Thus, sprays are used more often, and we preferred sprays to cotton pledgets in this study.

It is well known that lidocaine sprays have bad taste and smell, and that they anesthetize not only the nose but also the palate, nasopharynx, and throat. These features make lidocaine sprays uncomfortable for the patients $[5,6]$. On the other hand, decongestant sprays do not have these unfavorable effects [11]. Indeed, in this study, no differences were observed between oxymetazoline and placebo in terms of discomfort.

Interestingly, even though lidocaine is an anesthetic, it does not reduce pain during endoscopy $[5,11,19,20]$. In this study, we showed that pain scores were similar between lidocaine and placebo. In our opinion, the most important reason of pain during endoscopy with lidocaine is the congestion of turbinates. We reached this opinion with the evaluation of the physician's field of view. The field of view with lidocaine was narrower than with placebo. Moreover, while nasal decongestion was best achieved with oxymetazoline, the field of view decreased when oxymetazoline was used in combination with lidocaine. On the other hand, the best pain score was attained by lidocaine plus oxymetazoline. Therefore, the combined use of decongestant and lidocaine decreased pain compared to decongestant only. There- 
fore, the anesthetic effect of lidocaine could be obtained when turbinates are decongested.

Some physicians avoid using premedication for NE because of its systemic effects. Even though clinicians suppose that topical decongestants cause hypertension and reflex bradycardia, there are not many studies supporting this [7]. Similarly, NE may also increase pulse rate and arterial blood pressure [4]. However, as clinicians, we use decongestant sprays and perform endoscopies relatively often, but do not observe these changes as often as supposed. In this study, we showed that oxymetazoline slightly increased MBP 10 minutes after its application, but MBP returned to its initial level immediately after endoscopy. Interestingly, this effect of oxymetazoline was not seen when it was combined with lidocaine. We also showed that the pulse rate did not change significantly with any of the sprays used in this study. Furthermore, the changes of pulse rate and blood pressure due to the sprays and endoscopy were not clinically relevant. However, this finding may be a result of the exclusion of elders and patients with cardiovascular diseases. Therefore, we recommend using decongestants carefully in elderly patients and in patients with cardiovascular diseases. Nonetheless, these sprays can be used safely in young and healthy individuals.

The duration of endoscopic examination can also be an issue for physicians. In this regard, we evaluated the duration of endoscopy in order to consider the demands of physicians as well as of patients. This has not been considered in any of the similar studies so far. Our findings showed that the shortest duration of examination as well as the minimum pain score were provided by the decongestant-anesthetic combination. On the other hand, the best decongestion was obtained with decongestant alone. Standing on these observations, we suggest that duration of endoscopy is not only related to the wide passage in the nose but also strongly related to the patient's feeling of nasal pain.

In conclusion, the combined use of a decongestant and an anesthetic spray provided better field of view, reduced pain significantly, and decreased the duration of endoscopy. For these reasons, we considered decongestant plus anesthetic the best premedication method for nasal endoscopy. Because anesthetic sprays have bad taste and smell, we recommend using a decongestant spray alone in patients who refuse to use an anesthetic spray, while considering its side effects.

\section{CONFLICT OF INTEREST}

No potential conflict of interest relevant to this article was reported.

\section{ACKNOWLEDGMENTS}

We thank Gülhan Şimşek, the nurse of outpatient otolaryngolo- gy clinic of Erciyes University Medical School, for helping us collecting the data. This study was presented at International Otorhinolaryngology Head \& Neck Surgery Congress in Ankara,Turkey (April 17-19, 2014).

\section{REFERENCES}

1. Gaviola GC, Chen V, Chia SH. A prospective, randomized, doubleblind study comparing the efficacy of topical anesthetics in nasal endoscopy. Laryngoscope. 2013 Apr;123(4):852-8.

2. Midwinter KI,Ahmed A, Willatt D. A randomised trial of flexible versus rigid nasendoscopy in outpatient sinonasal examination. Clin Otolaryngol Allied Sci. 2001 Aug;26(4):281-3.

3. Nankivell PC, Pothier DD. Nasal and instrument preparation prior to rigid and flexible nasendoscopy: a systematic review. J Laryngol Otol. 2008 Oct;122(10):1024-8.

4. Ongkasuwan J, Yung KC, Courey MS. The physiologic impact of transnasal flexible endoscopy. Laryngoscope. 2012 Jun;122(6):13314.

5. Frosh AC, Jayaraj S, Porter G, Almeyda J. Is local anaesthesia actually beneficial in flexible fibreoptic nasendoscopy? Clin Otolaryngol Allied Sci. 1998 Jun;23(3):259-62.

6. Singh V, Brockbank MJ, Todd GB. Flexible transnasal endoscopy: is local anaesthetic necessary? J Laryngol Otol. 1997 Jul;111(7):6168.

7. Latham GJ, Jardine DS. Oxymetazoline and hypertensive crisis in a child: can we prevent it? Paediatr Anaesth. 2013 Oct;23(10):952-6.

8. Cain AJ, Murray DP, McClymont LG.The use of topical nasal anaesthesia before flexible nasendoscopy: a double-blind, randomized controlled trial comparing cophenylcaine with placebo. Clin Otolaryngol Allied Sci. 2002 Dec;27(6):485-8.

9. Georgalas C, Sandhu G, Frosh A, Xenellis J. Cophenylcaine spray vs. placebo in flexible nasendoscopy: a prospective double-blind randomised controlled trial. Int J Clin Pract. 2005 Feb;59(2):130-3.

10. Leder SB, Ross DA, Briskin KB, Sasaki CT. A prospective, doubleblind, randomized study on the use of a topical anesthetic, vasoconstrictor, and placebo during transnasal flexible fiberoptic endoscopy. J Speech Lang Hear Res. 1997 Dec;40(6):1352-7.

11. Shrestha BL, Amatya RC. Local anesthesia versus placebo in flexible nasopharyngo-laryngoscopy. J Rhinol-Otol. 2013;1:52-6.

12. Douglas R, Hawke L, Wormald PJ.Topical anaesthesia before nasendoscopy: a randomized controlled trial of co-phenylcaine compared with lignocaine. Clin Otolaryngol. 2006 Feb;31(1):33-5.

13. Johnson PE, Belafsky PC, Postma GN. Topical nasal anesthesia for transnasal fiberoptic laryngoscopy: a prospective, double-blind, cross-over study. Otolaryngol Head Neck Surg. 2003 Apr;128(4): 452-4.

14. Kasemsuwan L, Griffiths MV. Lignocaine with adrenaline: is it as effective as cocaine in rhinological practice? Clin Otolaryngol Allied Sci. 1996 Apr;21(2):127-9.

15. De Freitas RP, Hannah BC. Re: nasal and instrument preparation prior to rigid and flexible nasendoscopy: a systematic review. J Laryngol Otol. 2009 Oct;123(10):1188.

16. Sunkaraneni VS, Jones SE. Topical anaesthetic or vasoconstrictor preparations for flexible fibre-optic nasal pharyngoscopy and laryngoscopy. Cochrane Database Syst Rev. 2011 Mar;(3):CD005606.

17. Hu CT. Gauze pledgetting versus endoscopic-guided aerosolized spray for nasal anesthesia before transnasal EGD: a prospective, randomized study. Gastrointest Endosc. 2010 Jan;71(1):11-20.

18. Mishra P, Kaushik M, Dehadaray A, Qadri H, Raichurkar A, Seth T. Preparation of nose for nasal endoscopy: cotton pledget packing 
versus topical spray. A prospective randomized blinded study. Eur Arch Otorhinolaryngol. 2013 Jan;270(1):117-21.

19. Bonaparte JP, Javidnia H, Kilty S. A double-blind randomised controlled trial assessing the efficacy of topical lidocaine in extended flexible endoscopic nasal examinations. Clin Otolaryngol. 2011 Dec;
36(6):550-7.

20. Jonas NE, Visser MF, Oomen A, Albertyn R, van Dijk M, Prescott $\mathrm{CA}$. Is topical local anaesthesia necessary when performing paediatric flexible nasendoscopy? A double-blind randomized controlled trial. Int J Pediatr Otorhinolaryngol. 2007 Nov;71(11):1687-92. 